\title{
Reactive ion etching of poly(vinylidene fluoride-trifluoroethylene) copolymer for flexible piezoelectric devices
}

\author{
JIANG YongGang $^{1 *}$, SHIONO Syohei ${ }^{2}$, HAMADA Hiroyuki ${ }^{3}$, FUJITA Takayuki ${ }^{2,3}$, \\ ZHANG DeYuan ${ }^{1} \&$ MAENAKA Kazusuke ${ }^{2,3}$ \\ ${ }^{1}$ School of Mechanical Engineering and Automation, Beihang University, Beijing 100191, China; \\ ${ }^{2}$ Graduate School of Engineering, University of Hyogo, Himeji 671-2280, Japan; \\ ${ }^{3}$ Maenaka Human-Sensing Fusion Project, ERATO, Japan Science and Technology Agency, Himeji 671-2280, Japan
}

Received August 15, 2012; accepted September 12, 2012

\begin{abstract}
A microfabrication process for poly(vinylidene fluoride-trifluoroethylene) ( $\mathrm{P}(\mathrm{VDF}-\mathrm{TrFE})$ ) based flexible piezoelectric devices is proposed using heat controlled spin coating and reactive ion etching (RIE) techniques. Dry etching of $\mathrm{P}(\mathrm{VDF}-\mathrm{TrFE})$ in $\mathrm{CF}_{4}+\mathrm{O}_{2}$ plasma is found to be more effective than that using $\mathrm{SF}_{6}+\mathrm{O}_{2}$ or $\mathrm{Ar}+\mathrm{O}_{2}$ feed gas with the same radiofrequency power and pressure conditions. A maximum etching rate of $400 \mathrm{~nm} / \mathrm{min}$ is obtained using the $\mathrm{CF}_{4}+\mathrm{O}_{2}$ plasma with an oxygen concentration of $60 \%$ at an antenna power of $200 \mathrm{~W}$ and a platen power of $20 \mathrm{~W}$. The oxygen atoms and fluorine atoms are found to be responsible for the chemical etching process. Microstructuring of $\mathrm{P}(\mathrm{VDF}-\mathrm{TrFE})$ with a feature size of $10 \mu \mathrm{m}$ is achieved and the patterned films show a high remanent polarization of $63.6 \mathrm{mC} / \mathrm{m}^{2}$.
\end{abstract}

reactive ion etching, $\mathrm{P}(\mathrm{VDF}-\mathrm{TrFE})$, piezoelectric polymer, spin coating, flexible sensor

Citation: Jiang Y G, Shiono S, Hamada H, et al. Reactive ion etching of poly(vinylidene fluoride-trifluoroethylene) copolymer for flexible piezoelectric devices. Chin Sci Bull, 2013, 58: 2091-2094, doi: 10.1007/s11434-013-5836-9

Flexible microdevices varying from plastic solar cells, patchable body sensors and organic light-emitting diode (OLED) devices have been developed and are greatly improving the quality of life [1-3]. Piezoelectric flexible materials such as polyvinylidene fluoride (PVDF) and poly (vinylidene fluoride-trifluoroethylene) (P(VDF-TrFE)) have attracted great attention for flexible device applications. The piezoelectricity of PVDF was discovered by Kawai in 1969 [4], and there are at least four crystalline phases of PVDF, among which the polar phase I $(\beta)$ shows a very high piezoelectric voltage coefficient. P(VDF-TrFE), a copolymer of VDF and TrFE, shows a significant piezoelectricity with proper compositions. Due to their remarkable piezoelectric, mechanical and chemical properties, PVDF and P(VDFTrFE) are widely used for developing nanogenerators [5], metal-ferroelectric-metal capacitors [6], and heart beat sensors [7]. Cantilever shaped $\mathrm{P}(\mathrm{VDF}-\mathrm{TrFE})$ actuators were

*Corresponding author (email: jiangyg@ buaa.edu.cn) also fabricated for mini-robots application, in which a multilayered $\mathrm{P}(\mathrm{VDF}-\mathrm{TrFE})$ structure was proposed to achieve a large displacement with a low driving voltage [8].

Most of the PVDF based devices were fabricated using commercial PVDF films and traditional fabrication techniques such as scissoring, laser machining, and lamination $[9,10]$. With the aim of integrating piezoelectric polymers into microdevices, ionized evaporation, electrospray, electrospun, Langmuir-Blodgett deposition, and heat controlled spin coating methods were developed. Ionized evaporation and electrospray processes were reported to deposit PVDF films for pyroelectric infrared sensors in 1990s [11,12]. An electrospun method was employed to fabricate PVDF nanofibers for sensors and energy harvesters [5,13]. For fabrication of nonvolatile memory devices, low-temperature fabrication approaches including Langmuir-Blodgett deposition $[14,15]$ and spin coating methods [16] were developed. The spin coated $\mathrm{P}(\mathrm{VDF}-\mathrm{TrFE})$ films, intrinsically tending to form phase I crystal with a high remanent polarization, are 
widely used in recent researches $[6,17,18]$.

Microstructuring of the $\mathrm{P}(\mathrm{VDF}-\mathrm{TrFE})$ films is a critical process for fabrication of flexible piezoelectric devices. A modified Bosch process using $\mathrm{C}_{4} \mathrm{~F}_{8}, \mathrm{SF}_{6}$ and $\mathrm{O}_{2}$ gases were applied to $\mathrm{P}(\mathrm{VDF}-\mathrm{TrFE})$ etching with a commercial ion coupled plasma (ICP) reactive ion etching (RIE) equipment [8]. However, the RIE mechanism of P(VDF-TrFE) using fluorine or oxygen based gas has not been intensively studied. In this paper, we report the experimental study on the reactive ion etching mechanism of $\mathrm{P}(\mathrm{VDF}-\mathrm{TrFE})$ films for fabrication of $\mathrm{P}(\mathrm{VDF}-\mathrm{TrFE})$ based flexible microdevices.

\section{Experimental}

Figure 1 illustrates a schematic structure of $\mathrm{P}(\mathrm{VDF}-\mathrm{TrFE})$ based flexible devices, which comprises of a P(VDF-TrFE) layer sandwiched by double electrode layers on a flexible polydimethylsiloxane (PDMS) substrate. It can be fabricated using a metal deposition, $\mathrm{P}(\mathrm{VDF}-\mathrm{TrFE})$ spin coating and patterning process. Microfabrication of $\mathrm{P}(\mathrm{VDF}-\mathrm{TrFE})$ on the flexible PDMS substrate is a critical process for the batch fabrication of the P(VDF-TrFE) based devices.

The experiment on RIE of P(VDF-TrFE) is done with a procedure shown in Figure 2. A flexible PDMS substrate with a thickness of $300 \mu \mathrm{m}$ is used as the starting material. The surface of the PDMS substrate is modified by oxygen plasma to improve the adhesive strength between the PDMS substrate and the metal layer (Figure 2(a)). The adhesive strength is affected both by the chemical state of bonding and the morphology of the surface [20]. A layer of $\mathrm{Cr}-\mathrm{Au}$ with a thickness of $300 \mathrm{~nm}$ is deposited by sputtering as the bottom electrodes on the PDMS substrate. The P(VDFTrFE) layer is formed by the heat controlled spin-coat method (Figure 2(b)). As the composition of VDF and TrFE is very effective to achieve a high piezoelectric coefficient and a low current leakage, an optimized $\mathrm{P}(\mathrm{VDF}-\mathrm{TrFE})$ copolymer powder (KF W\#2200) with a composition of VDF/ TrFE=75/25 mol\% from KUREHA Corporation is used in our experiments. The $\mathrm{P}(\mathrm{VDF}-\mathrm{TrFE})$ powder is dissolved in methylethyl ketone with mechanical stirring at a temperature of $60^{\circ} \mathrm{C}$ to obtain a solution of $10 \mathrm{wt} \%$. After naturally cooling to room temperature, the solution is spin coated on the substrate at $1700 \mathrm{r} / \mathrm{min}$. The $2-\mu \mathrm{m}$-thick $\mathrm{P}(\mathrm{VDF}-\mathrm{TrFE})$ film is annealed at $140^{\circ} \mathrm{C}$ in nitrogen gas ambient for $2 \mathrm{~h}$ to enhance the crystallinity [21]. Aluminum is deposited

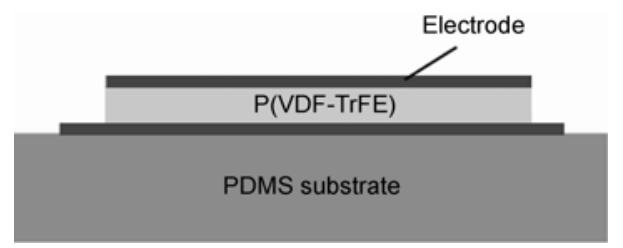

Figure 1 Schematic structure of $\mathrm{P}(\mathrm{VDF}-\mathrm{TrFE})$ based flexible sensing devices. (a)

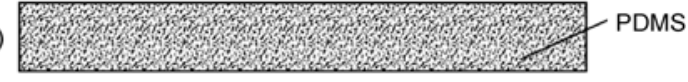

(b)

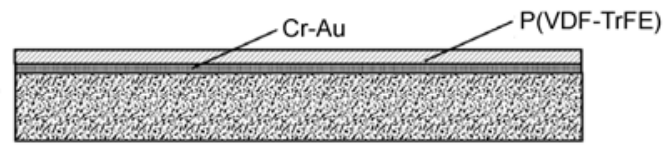

(c)

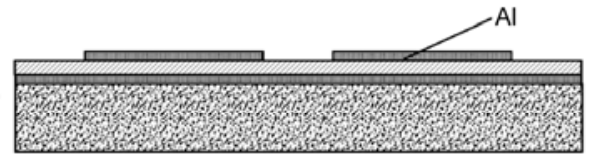

(d)

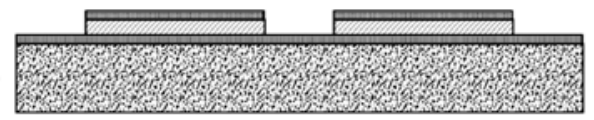

Figure 2 Experimental procedure for micromachining of $\mathrm{P}(\mathrm{VDF}-\mathrm{TrFE})$.

by sputtering with a thickness of $300 \mathrm{~nm}$ as the upper electrodes, and subsequently patterned by photolithography and wet etching processes (Figure 2(c)). Using the patterned aluminum as the mask, the $\mathrm{P}(\mathrm{VDF}-\mathrm{TrFE})$ is patterned by a home-made ICP-RIE system (Figure 2(d)).

\section{Results and discussion}

The X-ray diffraction (XRD) pattern is used to analyze the crystallinity of the $\mathrm{P}(\mathrm{VDF}-\mathrm{TrFE})$ film prepared by the heat controlled spin coating method as shown in Figure 3. The diffraction peak appeared at $20^{\circ}(2 \theta)$ is assigned to $\beta$-phase of the $\mathrm{P}(\mathrm{VDF}-\mathrm{TrFE})$ copolymer film. Dry etching experiments are carried out using the home-made RIE system. Antenna power refers to the radiofrequency (RF) power applied to the ICP antenna which is a conical coil, and platen power means the RF power applied to the cathode. Antenna power and gas composition are the most important factors that influence the etching results. As the result, we first investigate the antenna power dependence of the etching rates. In the experiment, pure oxygen is utilized as the feed gas with a flow rate of $50 \mathrm{sccm}$ at a process pressure of $5 \mathrm{~Pa}$ and a constant platen power of $20 \mathrm{~W}$. The etching rate

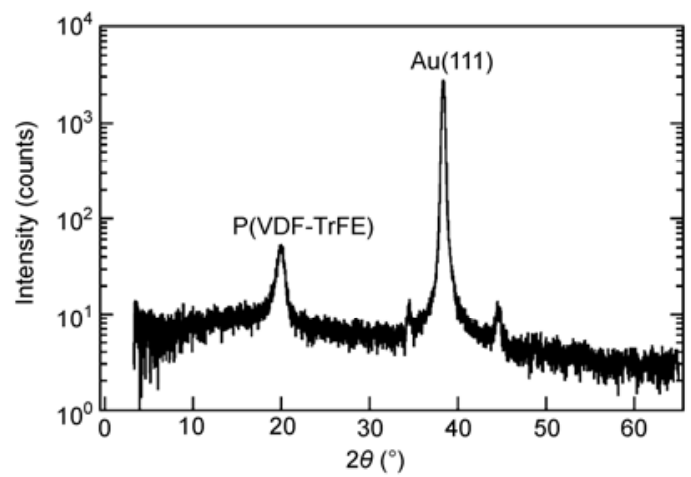

Figure 3 X-ray diffraction pattern of the $\mathrm{P}(\mathrm{VDF}-\mathrm{TrFE})$ film prepared by the heat controlled spin coating method. 
monotonously increases by improving the antenna power as shown in Figure 4. The high antenna power increases the density of reactive species, improving the etching rate of $\mathrm{P}(\mathrm{VDF}-\mathrm{TrFE})$.

In order to characterize the gas composition dependence of the etching rate, the RIE system is fed with $\mathrm{CF}_{4}+\mathrm{O}_{2}$, $\mathrm{SF}_{6}+\mathrm{O}_{2}$ and $\mathrm{Ar}+\mathrm{O}_{2}$ gas mixtures, respectively. The oxygen concentration varies from 0 to $100 \mathrm{vol} \%$. The experiment is done at a constant antenna power of $200 \mathrm{~W}$, a platen power of $20 \mathrm{~W}$, and a process pressure of $5 \mathrm{~Pa}$. With a total flow rate of $50 \mathrm{sccm}$, the etching rate variation with the gas composition is plotted in Figure 5. The etching rate using $\mathrm{SF}_{6}+\mathrm{O}_{2}$, or $\mathrm{Ar}+\mathrm{O}_{2}$ gas mixtures is monotonously increases by increasing the $\mathrm{O}_{2}$ concentration. As a result, it can be concluded that the oxygen is one of most effective species in the plasma responsible for the etching of $\mathrm{P}(\mathrm{VDF}-\mathrm{TrFE})$. However, there is an optimized composition of the $\mathrm{CF}_{4}+\mathrm{O}_{2}$ gas mixture to achieve the highest etching rate. It is well known that $\mathrm{CF}_{4}$ molecules are decomposed to $\mathrm{CF}_{2}^{+}$ions, $\mathrm{CF}_{3}{ }^{*}$ radicals, and fluorine atoms in the plasma, whilst oxygen exists in the form of oxygen atoms. Among the excited species such as $\mathrm{CF}_{3}{ }^{*}$ radicals, $\mathrm{CF}_{2}{ }^{+}$ions, and fluorine atoms, fluorine atom is responsible for reactive etching of $\mathrm{P}(\mathrm{VDF}-$ TrFE), as the etching rate is maximum when the density of fluorine atoms presents a maximum for P(VDF-TrFE) [22]. The following mechanism is suggested for the RIE process by fluorine and oxygen atoms.

$$
\begin{gathered}
{\left[\left(\mathrm{C}_{2} \mathrm{H}_{2} \mathrm{~F}_{2}\right) \mathrm{C}_{2} \mathrm{HF}_{3}\right]_{n}+\mathrm{F}+\mathrm{e} \rightarrow \mathrm{CF}_{2}^{+}+\mathrm{HF}+\mathrm{e}} \\
{\left[\left(\mathrm{C}_{2} \mathrm{H}_{2} \mathrm{~F}_{2}\right) \mathrm{C}_{2} \mathrm{HF}_{3}\right]_{n}+\mathrm{O}+\mathrm{e} \rightarrow \mathrm{CF}_{2} \mathrm{O}+\mathrm{CO}} \\
+\mathrm{CO}_{2}+\mathrm{HF}+\mathrm{e} \\
\mathrm{CF}_{2} \mathrm{O} \rightarrow \mathrm{CO}+2 \mathrm{~F}
\end{gathered}
$$

From above equations, it can be seen that the concentration of fluorine atoms can be improved by the addition of oxygen. The relative concentration of oxygen and fluorine atoms is responsible for the etching rate of $\mathrm{P}(\mathrm{VDF}-\mathrm{TrFE})$ when $\mathrm{CF}_{4}+\mathrm{O}_{2}$ feed gas mixture is utilized.

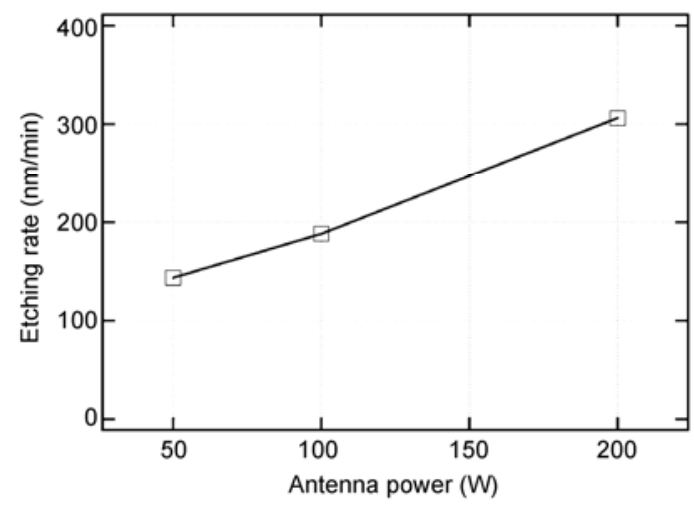

Figure 4 Etching rates of the P(VDF-TrFE) film as a function of antenna power using pure oxygen as the feed gas with a flow rate of $50 \mathrm{sccm}$ at a process pressure of $5 \mathrm{~Pa}$ and a constant platen power of $20 \mathrm{~W}$.

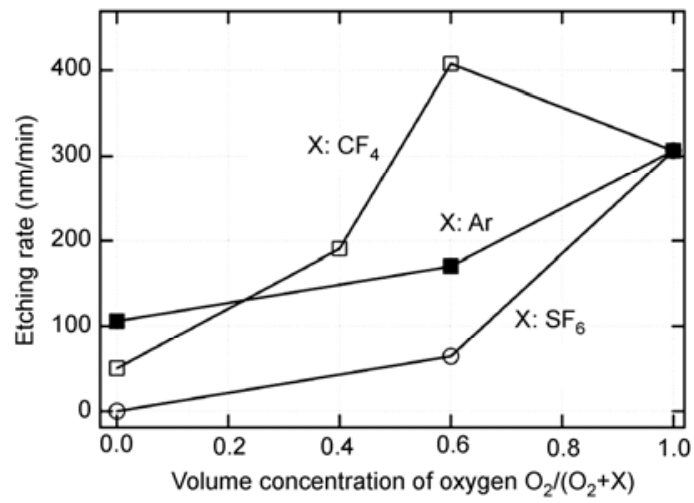

Figure 5 Etching rates variation with the feed gas compositions varying from $\mathrm{CF}_{4}+\mathrm{O}_{2}, \mathrm{Ar}+\mathrm{O}_{2}$, and $\mathrm{SF}_{6}+\mathrm{O}_{2}$ with a total flow rate of $50 \mathrm{sccm}$ at a process pressure of $5 \mathrm{~Pa}$, a constant platen power of $20 \mathrm{~W}$ and an antenna power of $200 \mathrm{~W}$.

$\mathrm{SF}_{6}$ is a well known reactive gas for plasma etching of silicon with an ultra low etching rate for polymers. The etching mechanism of $\mathrm{P}(\mathrm{VDF}-\mathrm{TrFE})$ is much different from that of PDMS. As there is silicon element in PDMS, plasma etching using the $\mathrm{SF}_{6}+\mathrm{O}_{2}$ gas mixture shows a higher etching rate than that using the $\mathrm{CF}_{4}+\mathrm{O}_{2}$ gas mixture, and the highest etching rate is obtained at a high $\mathrm{SF}_{6}$ percentage of 75 vol\% $[19,23]$. In order to realize the final piezoelectric flexible device, the specimen and concentration of feed gases should be optimized for selective etching of P(VDFTrFE) and PDMS.

Figure 6 illustrates the scanning electron microscope (SEM) image of the etching results. The $\mathrm{P}(\mathrm{VDF}-\mathrm{TrFE})$ strips with a feature size of $10 \mu \mathrm{m}$ have been successfully fabricated. In order to characterize the piezoelectric response of the $\mathrm{P}(\mathrm{VDF}-\mathrm{TrFE})$ films after being patterned by RIE, P-E hysteresis measurement is employed as shown in Figure 7. The remanent polarization and coercive electric field are found to be as high as $63.6 \mathrm{mC} / \mathrm{m}^{2}$ and $82.4 \mathrm{MV} / \mathrm{m}$, respectively. The successful etching of $\mathrm{P}(\mathrm{VDF}-\mathrm{TrFE})$ with excellent piezoelectricity using RIE can greatly contribute to the batch fabrication of P(VDF-TrFE) based flexible devices.

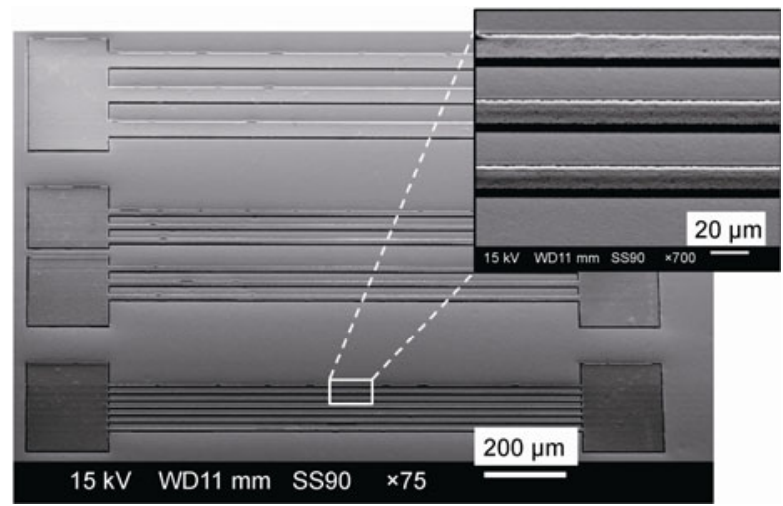

Figure 6 Scanning electron microscope (SEM) images of patterned $\mathrm{P}(\mathrm{VDF}-\mathrm{TrFE})$ microstructures. 


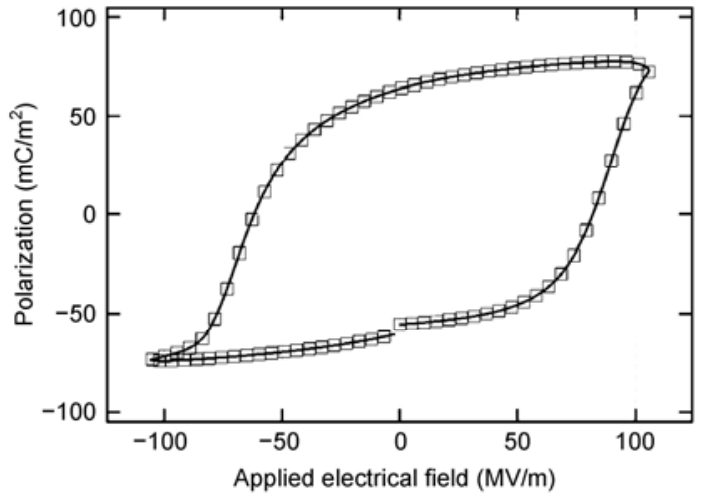

Figure 7 Measured P-E hysteresis curve of the patterned P(VDF-TrFE) film with a thickness of $2 \mu \mathrm{m}$.

\section{Conclusions}

Batch fabrication of $\mathrm{P}(\mathrm{VDF}-\mathrm{TrFE})$ based flexible devices was proposed. Micromachining of $\mathrm{P}(\mathrm{VDF}-\mathrm{TrFE})$ films with an excellent piezoelectric coefficient was achieved via the heat controlled spin coating and reactive ion etching methods. Gas mixtures of $\mathrm{CF}_{4}+\mathrm{O}_{2}, \mathrm{Ar}+\mathrm{O}_{2}$, and $\mathrm{SF}_{6}+\mathrm{O}_{2}$ were investigated to optimize the etching conditions. With an antenna power of $200 \mathrm{~W}$, a platen power of $20 \mathrm{~W}$, and a pressure $5 \mathrm{~Pa}$, a very high etching rate of $400 \mathrm{~nm} / \mathrm{min}$ was achieved with a mixture of $40 \% \mathrm{CF}_{4} / 60 \% \mathrm{O}_{2}$. It can be concluded that the excited fluorine and oxygen atoms are responsible for the reactive ion etching of P(VDF-TrFE) and the etching rate is related to their relative concentrations. The successful dry etching of $\mathrm{P}(\mathrm{VDF}-\mathrm{TrFE})$ will significantly contribute to batch fabrication of flexible piezoelectric devices.

The author would like to thank Mr. Iga and Mr. Hashimoto for helpful guidance in the reactive ion etching experiments and valuable discussions on the etching mechanism of $P(V D F-T r F E)$.

1 Brabec C J, Sariciftci N S, Hummelen J C. Plastic solar cells. Adv Funct Mater, 2001, 11: 15-26

2 Maenaka K, Fujita T, Hamada H, et al. Human sensing fusion for health and safety life. In: Dell J, Wlodarski W, Keating A, et al., eds. Proceedings of 5th Asia-Pacific Conference on Transducers and Micro-Nano Technology, Perth, Western Australia, 2010. 36-37

3 Tang C W, Vanslyke S A. Organic electroluminescent diodes. Appl Phys Lett, 1987, 51: 913

4 Kawai $\mathrm{H}$. The piezoelectricity of poly(vinylidene fluoride). Jpn J Appl Phys, 1969, 8: 975-976
5 Chang C, Tran Van H, Wang J, et al. Direct-write piezoelectric polymeric nanogenerator with high energy conversion efficiency. Nano Lett, 2010, 10: 726-731

6 Fujisaki S, Ishiwara H, Fujisaki Y. Low-voltage operation of ferroelectric poly(vinylidene fluoride-trifluoroethylene) copolymer capacitors and metal-ferroelectric-insulator-semiconductor diodes. Phys Lett, 2007, 90: 162902

7 Choi S, Jiang Z. A novel wearable sensor device with conductive fabric and PVDF film for monitoring cardiorespiratory signals. Sens Actuators A, 2006, 128: 317-326

8 Edqvist E, Snis N, Johansson S. Gentle dry etching of P(VDF-TrFE) multilayer micro actuator structures by use of an inductive coupled plasma. J Micromech Microeng, 2008, 18: 015007

9 Lee S, Bordachev E V, Zeman M J F. Femtosecond laser micromachining of polyvinylidene fluoride (PVDF) based piezo films. J Micromech Microeng, 2008, 18: 045011

10 Jiang Y, Shiono S, Hamada H, et al. Low-frequency energy harvesting using a laminated PVDF cantilever with a magnetic mass. In: Vullers R, Schaijk R V, Altena G, eds. Proceedings of PowerMEMS 2010, Leuven, Belgium, 2010. 375-378

11 Sakata J, Mochizuki M. Preparation of organic thin film by an electrospray technique I. Crystal forms and their orientation in poly (vinylidene fluoride) films. Thin Solid Films, 1991, 195: 175-184

12 Kubono A, Okui N. Polymer thin films prepared by vapor deposition. Prog Polym Sci, 1994, 19: 389-438

13 Hansen B J, Liu Y, Yang R, et al. Hybrid nanogenerator for concurrently harvesting biomechanical and biochemical energy. ACS Nano, 2010, 4: 3647-3652

14 Ducharme S, Bune A V, Blinov L M, et al. Critical point in ferroelectric Langmuir-Blodgett polymer films. Phys Rev B, 1998, 57: 2528

15 Palto S, Blinov L, Bune A, et al. Ferroelectric Langmuir-Blodgett films. Ferroelectr Lett Sect, 1995, 19: 65-68

16 Yamauchi N. A metal-insulator-semiconductor (MIS) device using a ferroelectric polymer thin film in the gate insulator. Jpn J Appl Phys, 1986, 25: 590-594

17 Naber R C G, Massolt J, Spijkman M, et al. Origin of the drain current bistability in polymer ferroelectric field-effect transistors. Appl Phys Lett, 2007, 90: 113509

18 Ramasundaram S, Yoon S, Kim K J, et al. Crystalline structure and ferroelectric response of poly(vinylidene fluoride) organically modified silicate thin films prepared by heat controlled spin coating. Macromol Chem Phys, 2009, 210: 951-960

19 Garra J, Long T, Currie J, et al. Dry etching of polydimethylsiloxane for microfluidic systems. J Vac Sci Technol A, 2002, 20: 975-982

20 Nakamura Y, Suzuki Y, Watanabe Y. Effect of oxygen plasma etching on adhesion between polyimide films and metal. Thin Solid Films, 1996, 290-291: 367-369

21 Zeng Z G, Zhu G D, Zhang L, et al. Effect of crystallinity on polarization fatigue of ferroelectric $\mathrm{P}(\mathrm{VDF}-\mathrm{TrFE})$ copolymer films. Chin $\mathrm{J}$ Polym Sci, 2009, 27: 479-485

22 Montazer-Rahamati P, Amouroux F A J. Etching of polypropylene (PE) and polyvinyldifluoride (PVDF) films by a low pressure discharge with corona configuration of electrodes in $\left(\mathrm{O}_{2}+\mathrm{CF}_{4}\right)$ mixtures. In: Ehlemann U, Lergon H G, Wiesemann K, eds. Proceedings of the 10th International Symposium on Plasma Chemistry (ISPC-10), Bochum, Germany, 1991. 2.2-5

23 Szmigiel D, Domanski K, Prokaryn P, et al. Deep etching of biocompatible silicone rubber. Microelectron Eng, 2006, 83: 1178-1181

Open Access This article is distributed under the terms of the Creative Commons Attribution License which permits any use, distribution, and reproduction in any medium, provided the original author(s) and source are credited. 